\title{
Label-Free Electrochemical DNA Detection Based on Electrostatic Interaction between DNA and Ferrocene Dendrimers ${ }^{\dagger}$
}

\author{
Ji Young Lee, Byung-Kwon Kim, Seongpil Hwang,",* Younghoon Lee, and Juhyoun Kwak* \\ Department of Chemistry, Korea Advanced Institute of Science and Technology (KAIST), Daejeon 305-701, Korea \\ ${ }^{*}$ E-mail: Juhyoun_Kwak@kaist.ac.kr \\ "Department of Chemistry, Myongji University, Yongin 449-728, Korea. ${ }^{*}$ E-mail: Shwang@mju.ac.kr \\ Received June 29, 2010, Accepted August 29, 2010
}

\begin{abstract}
A label-free DNA detection method was developed for a simple electrochemical DNA sensor with a short assay time. Self-assembled monolayers of peptide nucleic acid were used as a probe on gold electrodes. The formation of the self-assembled monolayers on the gold electrodes was successfully checked by means of cyclic voltammetry. The target DNA, hybridized with peptide nucleic acid, can be detected by the anodic peak current of ferrocene dendrimers, which interact electrostatically with the target DNA. This anodic peak current was measured by square wave voltammetry at $0.3 \mathrm{~V}$ to decrease the detection limit on the order of the nanomolar concentrations. As a result, the label-free electrochemical DNA sensor can detect the target DNA in concentrations ranging from $1 \mathrm{nM}$ to $1 \mu \mathrm{M}$ with a detection limit of $1 \mathrm{nM}$.
\end{abstract}

Key Words: Peptide nucleic acid, DNA detection, Ferrocene dendrimer, Label-free, Electrostatic interaction

\section{Introduction}

Deoxyribonucleic acid (DNA) sensing is important for disease diagnosis, single nucleotide polymorphisms, environmental analyses, and forensic applications. ${ }^{1-3}$ Optical methods based on surface plasmon resonance spectroscopies or fluorescence are the most widely used systems. ${ }^{3-5}$ Of all DNA detection methods, electrochemical methods have the advantages of low cost, simplicity, rapid response, and impressive compatibility with miniaturization. ${ }^{6-8}$ In electrochemical methods, hybridization is detected by a signal change that is mostly generated from redox labels such as $\left[\mathrm{Ru}\left(\mathrm{NH}_{3}\right)_{6}\right]^{3+2+2+6,7,9,10}\left[\mathrm{Fe}(\mathrm{CN})_{6}\right]^{4-13-}, 11-14$ $\left[\mathrm{Co}(\text { phen })_{3}\right]^{3+/ 2+15}$ methylene blue, ${ }^{16}$ ferrocene, ${ }^{8,17}$ and conductive polymers. ${ }^{2,4}$

A sandwich-type assay is widely used to detect the target DNA in electrochemical methods, especially in methods that involve the use of semiconductor nanoparticles, ${ }^{18}$ enzymes, ${ }^{19}$ and vesicles ${ }^{20}$ for labeling. However, because these types of assays need two-step hybridization and covalent modification of oligonucleotide with labels, the assay process is too difficult, inconvenient, and time-consuming for rapid field detection. ${ }^{2}$ Label-free DNA sensors offer an alternative approach that overcomes these disadvantages. Because label-free DNA sensors need single DNA hybridization, they can be used in a simple procedure of DNA sensor preparation. ${ }^{10}$

Recently, there has been an interest in DNA detection methods based on self-assembled monolayers (SAMs) of peptide nucleic acid (PNA). PNA is an analogue of DNA or ribonucleic acid (RNA). ${ }^{15}$ An artificially synthesized polymer, PNA has a similar structure to DNA or RNA except for its deoxyribose and ribose sugar backbone. The PNA backbone is made of repeating N-(2-aminoethyl)-glycine units linked by peptide bonds. Because the PNA backbone has a neutral charge, the binding

\footnotetext{
${ }^{\top}$ This paper is dedicated to Professor Hasuck Kim for his outstanding contribution to electrochemistry and analytical chemistry.
}

affinity between PNA and DNA strands is stronger than the affinity between DNA and DNA strands due to a deficiency of electrostatic repulsion. PNA appears to have a good binding affinity with complementary DNA. These characteristics are attributed to the chemical stability, which depends on the temperature and $\mathrm{pH}$ levels, as well as the biological stability and the specificity for a single-base pair mismatch. Moreover, the hybridization efficiency of PNA with complementary DNA is not affected by the salt concentration. When PNA on gold electrodes is hybridized with the target DNA, the bound target DNA can be detected by redox-active cations such as $\left[\mathrm{Ru}\left(\mathrm{NH}_{3}\right)_{6}\right]^{3+/ 2+}$ and $\left[\mathrm{Co}(\mathrm{phen})_{3}\right]^{3+2+}$, which bind with the negatively charged DNA by means of electrostatic attraction. ${ }^{6,7,9,10,15}$

A ferrocene dendrimer ( $\mathrm{Fc}-\mathrm{Den})$ is a redox-active cation that can perform multielectron-transfer. Because a dendrimer partially contains ferrocenes by covalent bonding, an Fc-Den can interact with the electrode via a redox reaction of ferrocenes. ${ }^{21-23}$ Ferrocenes in a dendrimer can amplify a signal whereas ferrocene molecules attached to oligonucleotide probes act as a redox tag because a single dendrimer contains multiple ferrocene molecules. ${ }^{17}$ Under a physiological condition where the $\mathrm{pH}$ is 7.4 , terminal protonated amine groups in a dendrimer have a positive charge due to the dendrimer's high $\mathrm{p} K_{\mathrm{a}}$ (of approximately 9.5). ${ }^{24}$ Therefore, an Fc-Den can bind to the target DNA by using electrostatic attraction under a physiological condition between the negatively charged DNA backbone and the terminated amine groups in the dendrimer. ${ }^{2}$ The trapped Fc-Den can transfer an electron with a electrode near the electrode surface that generates the electrochemical signal. ${ }^{26}$

We propose a label-free electrochemical DNA sensor: it uses PNA on gold and Fc-Den as a general marker. The hybridization of DNA with PNA is detected by the anodic peak current of an Fc-Den, which interacts electrostatically with the target DNA. The anodic peak current of the Fc-Den is measured by means of cyclic voltammetry (CV) and square wave voltammetry (SWV). 
The anodic peak current of the SWV provides a quantitative method of detecting the hybridization of DNA.

\section{Experimental Section}

Unless otherwise indicated, all reagents were obtained from Sigma or Aldrich and used as received. Ultra-pure water $(>18$ $\mathrm{M} \Omega$, Millipore) was used in all the experiments. A phosphate buffer (100 mM, pH 7.4) was prepared with sodium phosphate monobasic monohydrate and sodium phosphate dibasic heptahydrate. Phosphate-buffered saline (PBS) ( $\mathrm{pH} 7.4$ ) was prepared with $0.01 \mathrm{M}$ sodium phosphate, $0.138 \mathrm{M} \mathrm{NaCl}$, and $2.7 \mathrm{mM} \mathrm{KCl}$.

The PNA probe was synthesized by Panagene (Daejeon, Republic of Korea) and the sequence of PNA was HS-Cys-AEEATGT ACG TCA CAA CTA (from the $N$-terminus to the $C$-terminus). The HS-Cys of the PNA at the $N$-terminus is indicative of the anchoring cysteine group. AEEA stands for the (aminoethyl) ethoxy acetyl linker. The target DNA was obtained from Genotech (Daejeon, Republic of Korea). The sequence of the complementary-poly T DNA target was 5'-TTT TTT TTT TAG TTG TGA CGT ACA-3'. The sequence of the mismatched target DNA was 5'-CCA ACC ACA CCA ACC-3'. All the PNA and DNA oligonucleotides were purified by reversed-phase HPLC.

The Fc-Den was synthesized on the basis of previous studies. ${ }^{19,20}$ Briefly, the Fc-Den was prepared by the following principle: An amine-terminated G4 poly-(amidoamine) (PAMAM) dendrimer has 64 amine groups which can partially form covalent bonds with ferrocenecarboxaldehyde. We made a partially ferrocenyl-tethered PAMAM G4 dendrimer with 5\% primary amine modification.

Gold electrodes were prepared by electron beam evaporation of $40 \mathrm{~nm}$ of Ti and then $150 \mathrm{~nm}$ of gold on $\mathrm{Si}$ (100) wafers. The electrodes were cleaned in a piranha solution (1:3(v/v) $25 \%$ $\mathrm{H}_{2} \mathrm{O}_{2} / 75 \% \mathrm{H}_{2} \mathrm{SO}_{4}$ ) for $5 \mathrm{~min}$, rinsed with distilled water, and then dried with nitrogen gas. (Warning: piranha reacts violently with organics.) We constructed the electrodes for the DNA detection by immersing the electrodes in $1 \mu \mathrm{M}$ PNA aqueous solution for $3 \mathrm{~h}$, rinsing them with distilled water, immersing them in $1 \mathrm{mM}$ 6-mercapto-1-hexanol (MCH) aqueous solution for $1 \mathrm{~h}$, rinsing them again with distilled water, and finally drying them under a stream of nitrogen gas. For the hybridization of the target DNA and the PNA, we immersed electrodes layered with a mixture of PNA and $\mathrm{MCH}$ in $150 \mu \mathrm{L}$ of PBS solution containing the target DNA. The immersion was conducted at room temperature for $1 \mathrm{~h}$. After washing the electrodes with distilled water, we immersed the electrodes for $30 \mathrm{~min}$ in a phosphate buffer containing $10 \mu \mathrm{M} \mathrm{Fc-Dens} \mathrm{to} \mathrm{bind} \mathrm{the} \mathrm{Fc-Dens} \mathrm{to} \mathrm{the}$ target DNA by electrostatic interaction. The electrodes were then thoroughly rinsed with distilled water.

The electrochemical experiments were performed with a $\mathrm{CHI}$ 900B (CH Instruments, USA). A standard three-electrode cell was used with a modified gold working electrode, a Pt wire counter electrode, and an $\mathrm{Ag} / \mathrm{AgCl}$ reference electrode. The measurement was performed with PBS (pH 7.4). All cyclic voltammograms and square wave voltammograms were recorded at room temperature. The sweep of potential was scanned from $0 \mathrm{~V}$ to $+0.5 \mathrm{~V}$ at a scan rate of $50 \mathrm{mV} / \mathrm{s}$. The $\mathrm{SWV}$ was performed under the following conditions: an incremental poten- tial of $4 \mathrm{mV}$, an amplitude of $25 \mathrm{mV}$, and a frequency of $15 \mathrm{~Hz}$. Only the first scan was used in this experiment.

\section{Results and Discussion}

Fig. 1 shows a schematic diagram of label-free DNA detection with Fc-Dens and PNA. The PNA probe is immobilized on gold electrodes via the Au-S bond. MCH is then adsorbed on the gold electrodes. The adsorption reduces the nonspecific binding and causes the PNA probes to change from a prone position to an erect position for efficient hybridization with the target DNA. MCH effectively minimizes nonspecific interaction and enhances the hybridization because it has terminal hydroxyl groups and is shorter than the PNA linker. The target DNA is subsequently hybridized with the immobilized PNA as shown in Fig. 1(a). Finally, the target DNA binds with the FcDens, which acquire a positive charge from the electrostatic interaction (Fig. 1(b)). To provide a sufficient negative charge for the binding with the Fc-Dens, we designed the target DNA with extra poly $\mathrm{T}$ oligonucleotides. If the target DNA with the extra poly $\mathrm{T}$ oligonucleotides has a stronger negative charge, it may have a stronger electrostatic attraction to the positively charged Fc-Dens. We expect the Fc-Dens to interact electrostatically with the PNA-DNA double helix and to be wound by the overhang of the target DNA. These poly T extra sequences may reinforce the binding of the Fc-Dens with the target DNA. After the Fc-Dens are bound to the target DNA and then washed with distilled water, the Fc-Dens on the surface are measured electrochemically. The electron transfer between the Fc-Dens and the electrodes can be performed near the electrode surface. ${ }^{26}$ The redox current of the ferrocene can be effectively measured by electrochemical methods such as CV and SWV.

Fig. 2 shows the CV results for a redox reaction of $1 \mathrm{mM}$ of $\left[\mathrm{Fe}(\mathrm{CN})_{6}\right]^{4-13-}$ on the PNA-modified gold electrodes. In addition, the solid line (a) in the figure shows characteristic redox peaks for $1 \mathrm{mM}$ of $\left[\mathrm{Fe}(\mathrm{CN})_{6}\right]^{4-3^{-}}$on the bare gold electrodes. For the PNA-modified gold electrodes, the SAMs of the PNA slightly hinder the charge transfer. As shown by the dotted line (b) in Fig. 2, the peak-to-peak separation $\left(\Delta E_{\mathrm{p}}\right)$ is weakly broadened and the redox peak current of $\left[\mathrm{Fe}(\mathrm{CN})_{6}\right]^{4^{-/ 3^{-}}}$is diminished. When the PNA probe is hybridized with the target DNA, $\Delta E_{\mathrm{p}}$ is increased and the redox peak current is decreased (as shown by part (c) of Fig. 2). Because the negative charge of the target DNA

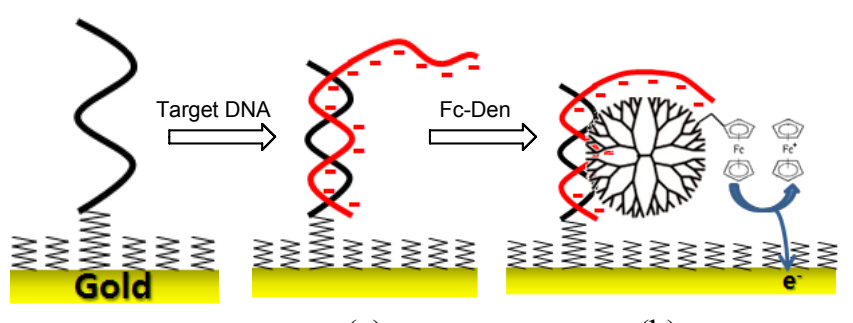

(a)

(b)

Figure 1. Schematic diagram of Fc-Den-based label-free DNA detection. (a) Hybridization of the target DNA with PNA. (b) Binding of Fc-Dens to the target DNA and the electrochemistry of Fc-Dens with the gold electrode. 


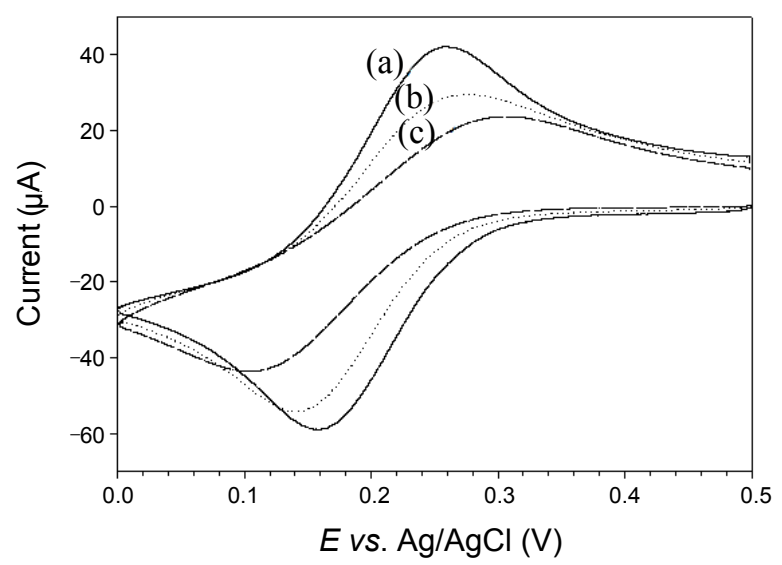

Figure 2. Cyclic voltammograms of $1 \mathrm{mM}$ of $\left[\mathrm{Fe}(\mathrm{CN})_{6}\right]^{4-/ 3-}$ on (a) bare gold electrodes $\left(\Delta E_{\mathrm{p}}=101 \mathrm{mV}\right)$, (b) PNA-modified gold electrodes $\left(\Delta E_{\mathrm{p}}=145 \mathrm{mV}\right)$, and (c) PNA/MCH/DNA-modified gold electrodes $\left(\Delta E_{\mathrm{p}}=203 \mathrm{mV}\right)$ at a scan rate of $50 \mathrm{mV} / \mathrm{s}$ in PBS (pH 7.4).

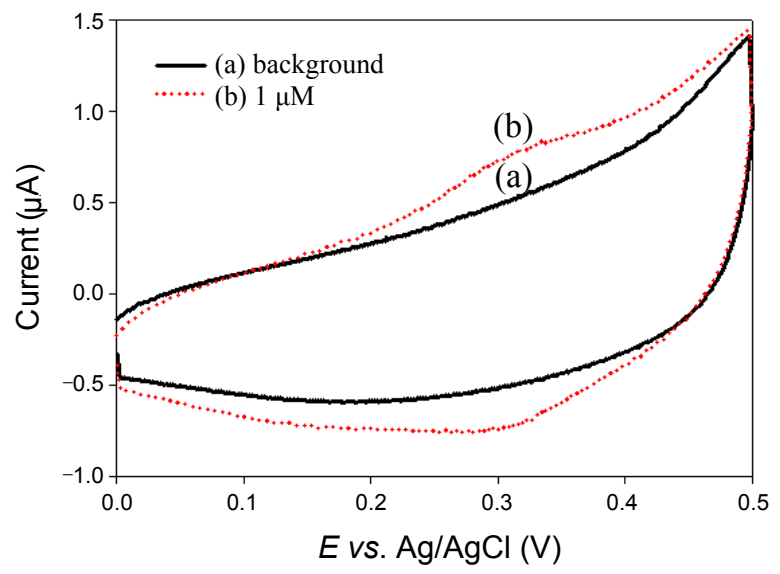

Figure 3. Cyclic voltammograms of Fc-Dens bound to the target DNA that is hybridized with a PNA probe on gold electrodes: (a) PNA/MCHmodified and (b) PNA/MCH/DNA-modified DNA $(1 \mu \mathrm{M})$ sensing electrodes at a scan rate of $50 \mathrm{mV} / \mathrm{s}$ in PBS (pH 7.4).

repels the negatively charged $\left[\mathrm{Fe}(\mathrm{CN})_{6}\right]^{4-/ 3-}$, there is greater interference in the charge transfer between $\left[\mathrm{Fe}(\mathrm{CN})_{6}\right]^{4-/ 3^{-}}$in the solution and the electrodes. These results demonstrate that PNA can be successfully immobilized on gold electrodes and retain the ability to be hybridized with DNA.

Fig. 3 shows the CV results for the redox reaction of surfacebound Fc-Dens on the PNA-modified gold electrodes. After the Fc-Dens are immobilized and washed with distilled water, they exhibit no redox current peak on the PNA/MCH-modified gold electrodes (part (a) of Fig. 3). This result indicates that the nonspecific binding of the Fc-Dens is negligible. When the Fc-Dens were immersed in an electrode PNA probe hybridized with the target DNA $(1 \mu \mathrm{M})$, the Fc-Den redox peak was about $0.3 \mathrm{~V}$. The anodic current of this redox peak is $0.75 \mu \mathrm{A}$ (part (b) of Fig. 3). Thus, the Fc-Dens can effectively bind to the target DNA via electrostatic interaction. However, this small Fc-Den redox peak current in the $\mathrm{CV}$ results is insufficient to distinguish the faradic current of the Fc-Dens from the nonfaradic current.

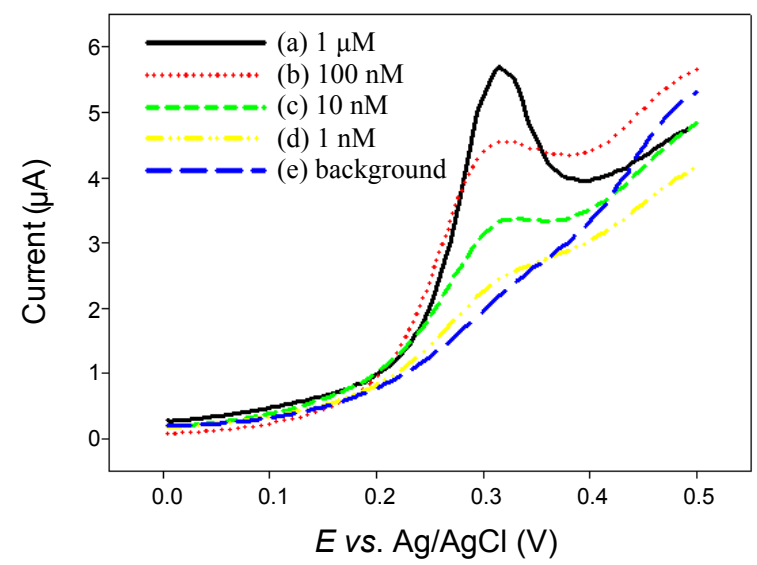

Figure 4. Square wave voltammograms of Fc-Dens bound to the target DNA hybridized with a PNA probe on gold electrodes: PNA/ MCH/DNA-modified ((a) $1 \mu \mathrm{M}$, (b) $100 \mathrm{nM}$, (c) $10 \mathrm{nM}$, and (d) $1 \mathrm{nM}$ ) and (e) PNA/MCH-modified DNA sensing electrodes in PBS (pH 7.4), with an incremental potential of $4 \mathrm{mV}$, an amplitude of $25 \mathrm{mV}$, and a frequency of $15 \mathrm{~Hz}$.

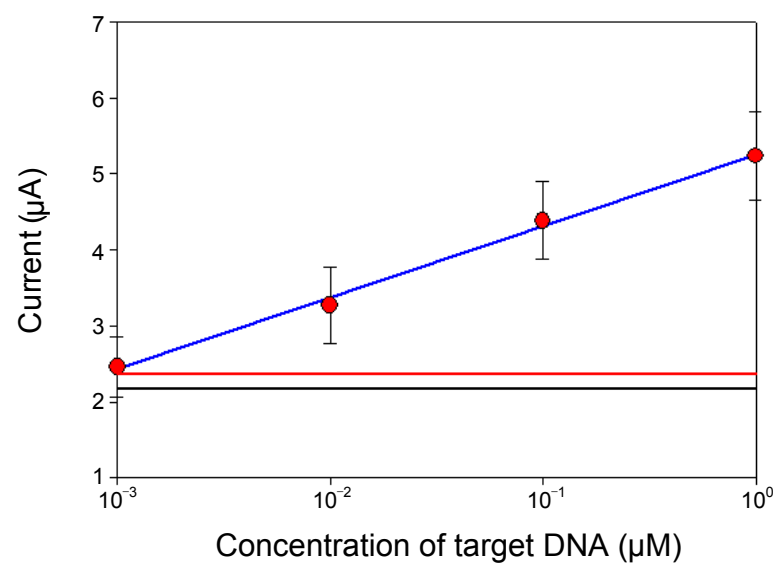

Figure 5. Calibration curve for the dependence of the anodic peak currents in square wave voltammograms at $0.3 \mathrm{~V}$ on target DNA concentrations. The black solid line corresponds to three times the standard deviation for zero target DNA. The red solid line corresponds to the nonspecific binding of the mismatched target DNA $(1 \mu \mathrm{M})$. Each point was measured at least three times. The other experimental conditions are the same as those described in the experimental section.

SWV was performed to minimize the capacitive charging current and decrease the detection limit on the order of nanomolar concentrations. Because of its ability to measure the current at the end of each potential change, SWV can confirm the presence of a minimized capacitive charging current. Thus, the enhanced current signal from the Fc-Den oxidation can be obtained for the purpose of lowering the detection limit. The SWV results in Fig. 4 show that the Fc-Dens bound to the target DNA have anodic peaks at around $0.3 \mathrm{~V}$; and these results are in good agreement with the $\mathrm{CV}$ results. The larger peaks are due to the minimization of the charging current. Thus, SWV is a more suitable electrochemical method for the signal transduction by Fc-Dens. These Fc-Den anodic peak currents in the square wave voltammograms seem to be proportional to the concentrations of the target DNA. As shown in Fig. 4, there is 
a weak anodic peak current at a DNA concentration of $1 \mathrm{nM}$ but no oxidation peak current for the background current. We can deduce, therefore, that the detection limit is about $1 \mathrm{nM}$ of the target DNA.

Fig. 5 shows a calibration curve of the dependence of the anodic peak currents in the square wave voltammograms at $0.3 \mathrm{~V}$ on various target DNA concentrations. Each concentration of the target DNA was measured at least three times. The anodic peak current at $1 \mathrm{nM}$ of the target DNA, $2.46 \mu \mathrm{A} \pm 0.40 \mu \mathrm{A}$ (mean \pm standard deviation), is higher than that of zero target DNA (background), $1.91 \mu \mathrm{A} \pm 0.27 \mu \mathrm{A}$ (mean \pm three times the standard deviation). Thus, the estimated detection limit of the DNA sensor is $1 \mathrm{nM}$. Moreover, the DNA sensor can detect the target DNA with concentrations ranging from $1 \mathrm{nM}$ to $1 \mu \mathrm{M}$. The mean data in Fig. 5 are in accord with the current values shown in Fig. 4 at $0.3 \mathrm{~V}$. We tested the nonspecific binding of the mismatched target DNA on the PNA-modified surfaces. The results confirm weak nonspecific binding of the mismatched target DNA $(1 \mu \mathrm{M})$. The numerical value is $2.33 \mu \mathrm{A} \pm 0.029 \mu \mathrm{A}$ (mean \pm standard deviation), and this value is lower than the signal of $1 \mathrm{nM}$ of the target DNA. This result means that the electrochemical responses originate from the specific interaction between PNA and DNA. The detection limit of our approach is higher than those of sandwich assays. However, our approach does not require any chemical modification or time-consuming amplification steps. Therefore, the use of Fc-Dens in our method is suitable for field work, especially when a high concentration target needs to be assayed urgently. In addition, recent developments in layer-by-layer technology suggest that multilayered Fc-Dens could enhance the detection limit.

\section{Conclusion}

We suggest a label-free electrochemical DNA sensor that uses PNA and Fc-Dens. The Fc-Dens can bind to the target DNA via electrostatic interaction. If the target DNA is hybridized with PNA, it can be estimated on the basis of the bound Fc-Den because the PNA probe has a neutral charge. The Fc-Den anodic peak currents in the square wave voltammograms seem to be proportional to the log concentrations of the target DNA in the range of $1 \mathrm{nM}$ to $1 \mu \mathrm{M}$. The detection limit of an Fc-Den labelfree electrochemical DNA sensor is $1 \mathrm{nM}$. This experiment provides information on the electrostatic interaction between Fc-Dens and the target DNA, and the results are potentially useful for the development of a label-free DNA detection method.

Acknowledgments. J. Kwak acknowledges the support from the Nano/Bio Science \& Technology Program (2010-0008213) of the Ministry of Education, Science and Technology. The work is also partially supported by a grant from the Korea Research Foundation, which is funded by the Korean Government (F01-2008-000-10153-0). S. Hwang acknowledges the support from the 2008 Research Fund of Myongji University and the Basic Science Research Program of the National Research Foundation of Korea, which is funded by the Ministry of Education, Science and Technology (2010-0015442).

\section{References}

1. Drummond, T. G.; Hill, M. G.; Barton, J. K. Nat. Biotechnol. 2003, 21,1192 .

2. Zhang, L.; Sun, H.; Li, D.; Song, S.; Fan, C.; Wang, S. Macromol. Rapid Commun. 2008, 29, 1489.

3. Sassolas, A.; Leca-Bouvier, B. D.; Blum, L. J. Chem. Rev. 2008 , $108,109$.

4. Reisberg, S.; Dang, L. A.; Nguyen, Q. A.; Piro, B.; Noel, V.; Nielsen, P. E.; Le, L. A.; Pham, M. C. Talanta 2008, 76, 206.

5. Homola, J.; Yee, S. S.; Gauglitz, G. Sens. Actuators, B Chem. 1999, 54,3 .

6. Fang, Z.; Kelley, S. O. Anal. Chem. 2009, 81, 612.

7. Steichen, M.; Decrem, Y.; Godfroid, E.; Buess-Herman, C. Biosens. Bioelectron. 2007, 22, 2237.

8. Won, B. Y.; Yoon, H. C.; Park, H. G. Analyst 2008, 133, 100.

9. Cheng, A. K. H.; Ge, B.; Yu, H.-Z. Anal. Chem. 2007, 79, 5158.

10. Das, J.; Lee, J.-A.; Yang, H. Langmuir 2010, 26, 6804.

11. Degefa, T. H.; Kwak, J. J. Electroanal. Chem. 2008, 612, 37.

12. Aoki, H.; Umezawa, Y. Electroanalysis 2002, 14, 1405.

13. Aoki, H.; Bühlmann, P.; Umezawa, Y. Electroanalysis 2000, 12 , 1272.

14. Yu, D.; Kim, K. Bull. Korean Chem. Soc. 2009, 30, 955.

15. Wang, J.; Palecek, E.; Nielsen, P. E.; Rivas, G.; Cai, X.; Shiraishi, H.; Dontha, N.; Luo, D.; Farias, P. A. M. J. Am. Chem. Soc. 1996, $118,7667$.

16. Kelly, S. O.; Barton, J. K. Bioconjugate Chem. 1997, 8, 31.

17. Kang, D.; Zuo, X.; Yang, R.; Xia, F.; Plaxco, K. W.; White, R. J. Anal. Chem. 2009, 81, 9109.

18. Das, J.; Aziz, Md. A.; Yang, H. J. Am. Chem. Soc. 2006, 128, 16022.

19. Alfonta, L.; Singh, A. K.; Willner, I. Anal. Chem. 2001, 73, 91.

20. Patolsky, F.; Lichtenstein, A.; Willner, I. J. Am. Chem. Soc. 2000, $122,418$.

21. Yoon, H. C.; Hong, M.-Y.; Kim, H.-S. Anal. Chem. 2000, 72, 4420.

22. Kim, E.; Kim, K.; Yang, H.; Kim, Y. T.; Kwak, J. Anal. Chem. 2003, 75, 5665 .

23. Kim, J. M.; Ju, H.; Choi, H. S.; Lee, J.; Kim, J.; Kim, J.; Kim, H. D.; Kim, J. Bull. Korean Chem. Soc. 2010, 31, 491.

24. Yoon, H. C.; Lee, D.; Kim, H.-S. Anal. Chim. Acta 2002, 456, 209.

25. Priyadarshy, S.; Risser, S. M.; Beratan, D. N. J. Biol. Inorg. Chem. 1998, 3, 196.

26. Kwon, S. J.; Yang, H.; Jo, K.; Kwak, J. Analyst 2008, 133, 1599. 